\title{
Soft X-ray Scattering and Halos from Dust
}

\author{
Randall K. Smith ${ }^{1}$ and Eli Dwek \\ Code 685 \\ NASA Goddard Space Flight Center \\ Greenbelt, MD 20771
}

\begin{abstract}
Small angle scatterings of X-rays by interstellar dust particles create halos around $\mathrm{x}$-ray sources. The halo intensity and its projected radial distribution around a source can provide important information on the spatial distribution of the dust along the line of sight to the source, and on the physical properties of the scattering dust particles. Halos around x-ray point sources have been used by several authors to infer that the scattering dust particles are fluffy in nature, consisting of aggregates of smaller refractory particles with vacuum occupying a significant fraction of their volume.

The nature and morphology of interstellar dust particles has recently gained new importance, since fluffy, composite dust particles have been suggested as a possible solution to the interstellar carbon "crisis." This crisis results from the discrepancy between the abundance of carbon in the interstellar medium available for creating dust, and the significantly larger amount of carbon that must be in dust in order to account for the UV-optical interstellar extinction in the diffuse ISM.

Previous studies of x-ray scattering have used the Rayleigh-Gans (RG) approximation to the differential scattering cross section for calculating halo properties. However, the validity of the RG approximation fails for energies below $1 \mathrm{keV}$. We use the exact Mie solution for the differential scattering cross section and find that for these energies the scattering becomes much less efficient than is predicted by the RG approximation. Furthermore, the effects of $\mathrm{K}$ and $\mathrm{L}$ band absorption by atoms in the dust become important. The net effect is that the RG approximation systematically and substantially overestimates the intensity of the halo below $1 \mathrm{keV}$, relative to the Mie solution result. In particular Mathis et al. (1995) used the weaker-than-expected halo intensity observed around Nova Cygni 1992 to conclude that interstellar dust must be fluffy. Using the Mie solution to the scattering intensity and including the effects of absorption, we find that, contrary to the conclusion of Mathis et al. (1995), the halo around Nova Cygni 1992 does not require interstellar dust grains to be fluffy in nature, and that the data is consistent with scattering from a mix of bare refractory silicate and carbon grains as well.
\end{abstract}

\footnotetext{
${ }^{1}$ present address: Smithsonian Astrophysical Observatory, MS 70, 60 Garden Street, Cambridge, MA 02138; email: rsmith@cfa.harvard.edu
} 
Subject headings: ISM: dust, extinction, abundances - X-rays: General, ISM — stars: Individual (Nova V1974 Cygni; Nova Cygni 1992)

\section{Introduction}

Measuring the small-angle scattering of X-rays from particles embedded in a uniform medium to calculate their size and shape has long been a standard technique in microbiology (Glatter \& Kratky, 1982). The scattering of X-rays from dust grains in the interstellar medium (ISM) was first considered by Overbeck in 1965. However, the weak x-ray halo generated by small-angle x-ray scattering from dust has only recently become observable (Mauche \& Gorenstein, 1989; Mathis et al., 1995; Predehl \& Klose, 1996). In the laboratory, small-angle x-ray scattering observations can measure the size and shape of the scattering particles quite accurately, but astrophysical applications are still sharply limited by the availability of sufficiently bright x-ray sources and the spatial and energy resolution of x-ray telescopes. However, recent observations using the ROSAT Position Sensitive Proportional Counter (PSPC) (Predehl \& Schmitt, 1995) have found x-ray halos around 25 point sources and 4 supernova remnants, using the moderate $25^{\prime \prime}$ resolution of this instrument. The Advanced X-ray Astrophysical Facility (AXAF) telescope will have $\sim 1^{\prime \prime}$ resolution (Canizares, 1990), and should dramatically increase the number of sources with visible halos.

In general the halo formed around an x-ray point source by small-angle scattering depends, in addition to the spatial distribution of the dust, upon many details of the interstellar dust grain population. The intensity varies linearly with the number of grains along the line of sight, and is highly dependent upon the size distribution of the larger grains, which more efficiently scatter X-rays. An individual spherical dust grain scatters X-rays with a angular distribution determined by the grain size distribution, composition, and density. Given the dust parameters, the spatial distribution of the grains can be estimated by comparing this scattered distribution with the observed halo. Although it is difficult to extract each dust grain parameter separately, measurements of the halo's intensity as a function of energy and angle from the source can provide useful limits for the allowable dust grain model parameters.

The general theory of x-ray scattering by dust grains has been discussed in detail by Mauche \& Gorenstein (1986) (MG86) and Mathis \& Lee (1991) (ML91). Their results were based on the Rayleigh-Gans (RG) approximation to the differential scattering cross section. Predehl \& Klose (1996)(PK96) discussed the possibilities for extracting dust grain parameters by observing x-ray halos with current x-ray telescopes. They also considered the limitations of the Rayleigh-Gans approximation and the effects of absorption in the dust grain on the scattered X-rays. Recent observations of x-ray halos (Mathis et al., 1995; Woo et al., 1994; and Predehl, 1996), using both the ROSAT and $A S C A$ telescopes, have found that the observed halo intensity is less than that 
predicted by the general theory using the RG approximation. These authors concluded that their results were evidence for "fluffy" dust grains which contain many holes and voids.

These results are of particular interest because of the carbon "crisis" in the interstellar medium (ISM) (Cardelli et al., 1996). Observations have suggested that the amount of carbon available to be in dust grains is substantially less than the amount necessary to make the grains which account for the observed extinction. Mathis (1996; M96) has suggested that fluffy dust grains would account for the extinction measurements while reducing the amount of carbon necessary to be locked up in the dust.

However, Dwek (1997) pointed out that the composite fluffy dust model (CFD) suggested by Mathis fails to resolve the interstellar carbon crisis for several reasons: (1) the visual albedo of the CFD particles is lower than the observed one. The increased absorptivity of the composite dust grains produces therefore an excess of infrared emission over that observed with the Diffuse Infrared Background Experiment (DIRBE) and Far Infrared Absolute Spectrophotometer (FIRAS) instrument on board the Cosmic Background Explorer (COBE) satellite (Dwek et al., 1997); (2) The CFD model does not include polycyclic aromatic hydrocarbons (PAHs) as a grain constituent. As a result it does not take the abundance of carbon locked up in PAHs or the effect of PAHs on the UV and optical extinction into account. In light of these difficulties we re-examine the theory of x-ray scattering by interstellar dust particles and the evidence for the existence of composite fluffy dust particles in the ISM.

We present in $\S 2$ a brief review of the theory, describing the Rayleigh-Gans ( $R G$ ) approximation to the x-ray scattering from a spherical particle and examining the range of its validity. In $\S 3$ we present the exact Mie solution to light scattering from a spherical particle, and describe the differences between the Mie theory and the Rayleigh-Gans approximation for various astrophysical situations, including the importance of absorption of X-rays in the dust. In $\S 4$ we apply the Mie theory to the observations of Nova Cygni 1992 from Mathis et al. (1995), and discuss the implications for dust models.

\section{Basic theory}

The physical situation we consider is the small-angle scattering of X-rays emitted by a bright x-ray source from dust grains in the ISM near the line of sight (LOS), which will create an x-ray halo around the point source. The scattered angles are small enough, that for astronomical purposes, we can assume that the path traveled by the unscattered primary X-rays is nearly identical to the paths of scattered photons, in terms of their distances and environments. We discuss this assumption and its limitations in Appendix A.

The x-ray source S (see Figure 1) at distance D from the observer O has an observed flux $F_{X}$ and spectrum $S(E)$ in the $\left\{E_{\min }, E_{\max }\right\}$ energy range, where $S(E)$ is normalized to unity over that interval. The emitted X-rays, traveling though the ISM scatter from a population of 


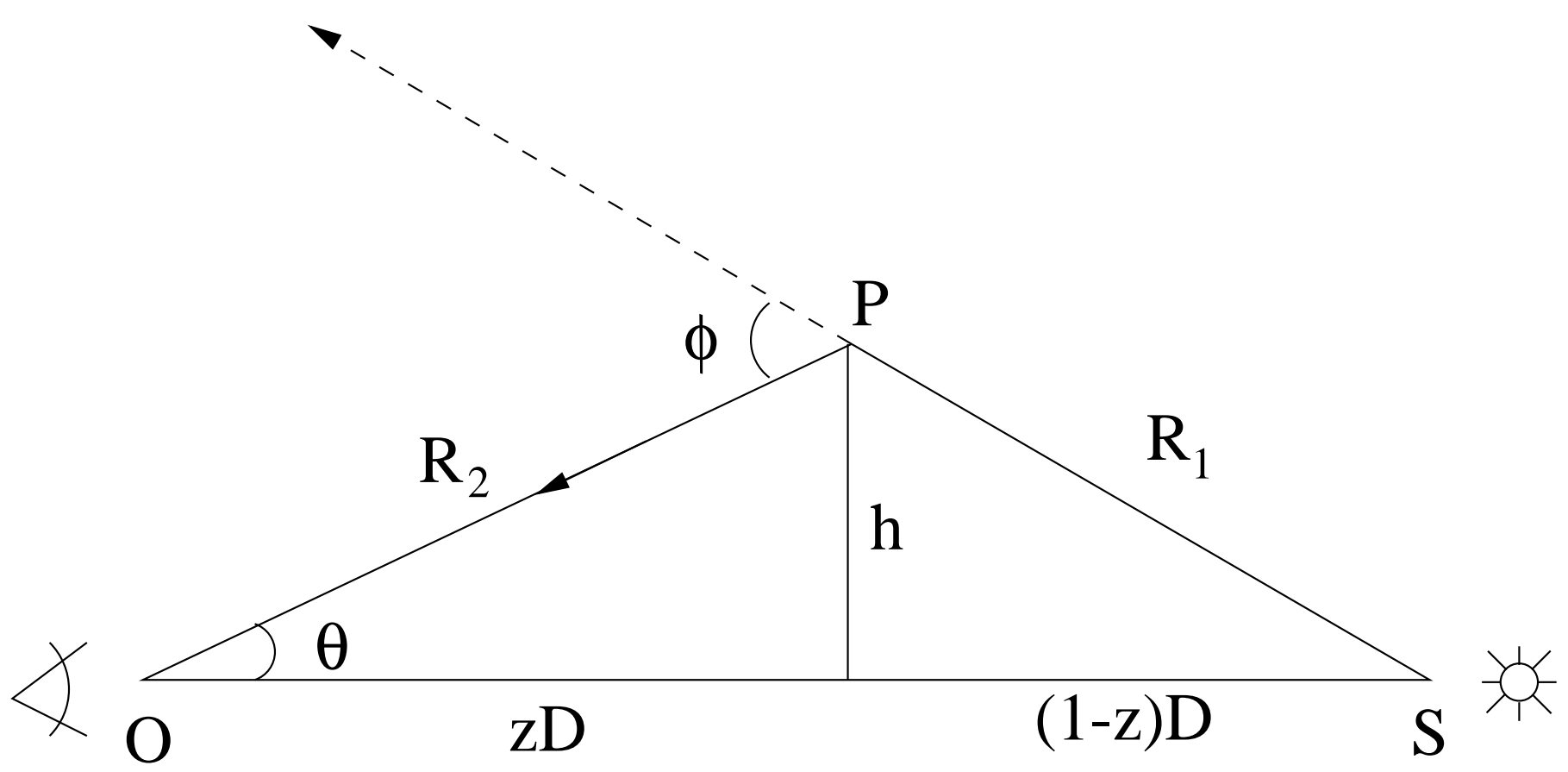

Fig. 1. - Scattering diagram of an X-ray emanating from a source $\mathrm{S}$ that is scattered through an angle $\phi$ by a dust particle located at $\mathrm{P}$ towards an observer $\mathrm{O}$. The parameter $z \equiv R_{2} \cos \theta / D$ is a dimensionless quantity denoting the projected distance to the dust particle from $\mathrm{O}$ along the line of sight to $\mathrm{S}$.

dust grains with number density $n(a) d a$ per $\mathrm{H}$ atom in the interval $\{a, a+d a\}$ for grain radii in the range $\left\{a_{\min }, a_{\max }\right\}$. We assume that the grain size distribution is uniform but that its total number density can vary along the LOS. We characterize the spatial distribution of the grains by the function $f(z)$ where $z \equiv R / D$ is the normalized distance from the observer for an actual distance $R$ from the observer; $f(z)$ is normalized to unity over the range $\{0,1\}$ Finally, the scattered power in a given direction is determined by the differential scattering cross section $(d \sigma / d \Omega)$, which is a function of x-ray energy, dust grain size, and scattering angle.

In the most general form, we can write the equation for the scattered intensity at angle $\theta$, in units of photons per $\mathrm{cm}^{2}$ per second per steradian as (see Appendix A):

$$
I_{s c a}(\theta)=\int_{E_{\min }}^{E_{\max }} d E F_{X} S(E) \int_{a_{\min }}^{a_{\max }} d a \mathrm{~N}_{\mathrm{H}} n(a) \int_{0}^{1} \frac{f(z)}{(1-z)^{2}}\left(\frac{d \sigma(E, a, \phi)}{d \Omega}\right) d z .
$$

Equation (11) apparently diverges for scattering near the source, where $z \rightarrow 1$. However, near the source the contribution to the flux scattered to the observer comes from relatively large scattering angles, for which $(d \sigma / d \Omega)$ is vanishingly small. So in practice using an upper limit $1-\epsilon$ instead of 1 to the position integral for some small $\epsilon$ is acceptable, since the integral from $1-\epsilon$ to 1 is negligible. Numerical experiments, with smoothly distributed dust $(f(z) \equiv 1)$, a single grain size, 
and a single x-ray energy, show that the integral (using the Mie solution for the scattering term) converges to within 1 part in $10^{6}$ when $\epsilon \equiv \theta$ (measured in radians). This conclusion holds for dust sizes in the range $0.1-1.0 \mu \mathrm{m}$ and x-ray energies in the range $0.1-1.0 \mathrm{keV}$. Using this result, we can modify equation (11) by changing the upper limit on $z$ to be $1-\theta$ so that:

$$
I_{s c a}(\theta)=F_{X} \mathrm{~N}_{\mathrm{H}} \int_{E_{\min }}^{E_{\max }} d E S(E) \int_{a_{\min }}^{a_{\max }} d a n(a) \int_{0}^{1-\theta} \frac{f(z)}{(1-z)^{2}}\left(\frac{d \sigma}{d \Omega}\right) d z .
$$

which removes the numerical difficulties.

In addition, in this paper we consider only singly-scattered X-rays. Although ML91 developed the theory of multiply-scattered x-ray halos, the brightest x-ray halos occur when the optical depth to scattering is low, $\tau_{s c a} \sim 0.2$ (PK96). PK96 also found that when the optical depth for scattering was high enough that multiple scattering was important, absorption along the LOS greatly reduced both the source and halo intensity.

\subsection{Rayleigh-Gans Theory}

The Rayleigh-Gans approximation for the differential scattering cross section $(d \sigma / d \Omega)$ requires the following two assumptions. First, that the reflection from the surface of the dust particle is negligible, i.e. that $|m-1| \ll 1$, where $m$ is the complex index of refraction of the dust. This ensures that the X-ray enters the dust particle instead of being reflected. The second, more stringent requirement is that

$$
k_{0} a|m-1| \ll 1
$$

where $k_{0}$ is the wave number of the X-ray and $a$ the radius of the grain. This ensures that the phase of the incident wave is not shifted inside the medium. We will show how, in this limit and for sufficiently small scattering angles, it is possible for the waves scattered throughout the dust grain to add coherently. In this situation, the intensity of the scattered waves is proportional to the number of scattering sites squared; i.e.:

$$
I \propto N^{2} \propto \rho^{2} a^{6}
$$

where $\rho$ is the mass density of the dust grain, and $a$ its radius.

A useful expression for the RG approximation assumptions can be derived using the "Drude approximation" to $|m-1|$ for a collection of free electrons responding to an oscillating external field. The Drude approximation (Bohren \& Huffman, 1983, p. 253) is

$$
m \approx \sqrt{1-\frac{\omega_{p}^{2}}{\omega^{2}+i \gamma \omega}}
$$

where $\omega$ is the circular frequency of the wave, $\omega_{p}^{2}=4 \pi c^{2} n_{e} r_{e}$ is the plasma frequency of the medium (for electron number density $n_{e}$, and where $r_{e}$ is the classical radius of the electron). 
The $\gamma$ term is a damping term due to electronic interactions with impurities or lattice vibrations. Assuming $|m-1| \ll 1$, we can rewrite (5) to get

$$
|m-1| \approx \frac{n_{e} r_{e} \lambda^{2}}{2 \pi}\left(\frac{1}{1+\frac{i \gamma}{\omega}}\right)
$$

where $n_{e}$ is the density of electrons, $r_{e}$ is the classical radius of the electron, and $\lambda$ the wavelength of the photon. Combining (6) with (3), and ignoring damping (i.e., $\gamma=0$ ) we get:

$$
a n_{e} r_{e} \lambda \ll 1 \text {. }
$$

Equation (7) can be expressed in terms of the dust mass density $\rho$ by assuming that the atomic constituents of the dust have one electron per 2 nucleons, so $n_{e}=\rho /\left(2 m_{H}\right)$. The coherency requirement for the validity of the $\mathrm{RG}$ approximation then becomes:

$$
\frac{a_{\mu \mathrm{m}}}{E_{k e V}}\left(\frac{\rho}{3 \mathrm{~g} \mathrm{~cm}^{-3}}\right) \ll 0.316 .
$$

A "rule of thumb" for normal interstellar dust grains is that the RG approximation is valid only if the energy of the $\mathrm{x}$-ray in $\mathrm{keV}$ is significantly larger than the grain radius in $\mu \mathrm{m}$.

Within this limit, the RG approximation completely describes the scattering of photons in a simple analytic form. The exact RG approximation for the differential scattering cross section is given in MG86, and shown below. The exact result is in terms of the spherical Bessel function $j_{1}$, but can be well-approximated by a Gaussian function:

$$
\begin{aligned}
\left(\frac{d \sigma}{d \Omega}\right)(E, a, \phi) & =2 a^{2}\left(\frac{2 \pi a}{\lambda}\right)^{4}|m-1|^{2}\left(\frac{j_{1}(y)}{y}\right)^{2}\left(1+\cos ^{2} \phi\right) \\
& \approx 2 a^{2}\left(\frac{2 \pi a}{\lambda}\right)^{4}|m-1|^{2} \frac{1}{9} \exp \left(-\frac{y^{2}}{2 \sigma^{2}}\right)\left(1+\cos ^{2} \phi\right)
\end{aligned}
$$

where $y=(4 \pi a / \lambda) \sin (\phi / 2)$ and $\sigma$ is a function of the energy and size of the dust particle. We see here the $a^{6}$ dependence described earlier; the $\rho^{2}$ dependence is implicit in the $|m-1|^{2}$ term (see eq. 6).

Insight into the mechanism and the maximum angle for coherent scattering can be derived from the fundamental physics of the scattering. Photons scatter from charged particles, and the differential scattering cross section for photons interacting with a collection of charged free particles is given by Jackson (1975) (eq. 14.111):

$$
\frac{d \sigma}{d \Omega}=\left|\sum_{j} \frac{e_{j}^{2}}{m_{j} c^{2}} e^{i \vec{q} \cdot \overrightarrow{r_{j}}}\right|^{2}\left|\vec{\epsilon}^{*} \cdot \overrightarrow{\epsilon_{o}}\right|
$$

where the sum is taken over all the charges in the medium. Here $e_{j}$ is the charge of the $j$ th particle, $m_{j}$ is its mass, and $r_{j}$ is its position relative to the center of the dust particle (see Figure 2). We define $\vec{q} \equiv\left(\omega \hat{n_{s}}-\omega_{0} \hat{n_{i}}\right) / c$, where $\hat{n_{i}}$ and $\hat{n_{s}}$ are unit vectors in the initial and scattered directions 


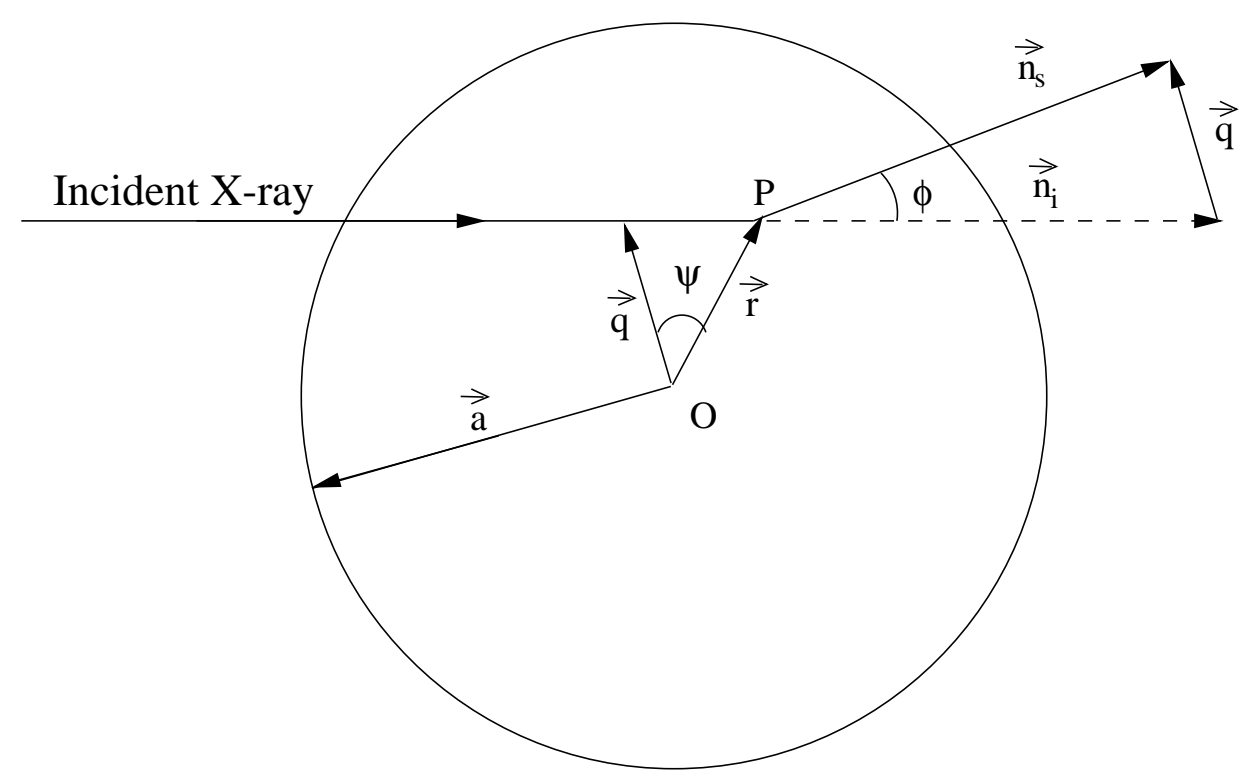

Fig. 2.- An X-ray traveling in direction $\overrightarrow{n_{i}}$ impinging upon a dust particle of radius $a$, interacting at a point $\mathrm{P}$ located at a distance $r$ from the center of the particle $\mathrm{O}$, and scattering in the direction $\overrightarrow{n_{s}}$.

respectively, $\omega_{0}$ and $\omega$ are the circular frequencies of the X-ray outside and inside the medium respectively, and $c$ is the speed of light. The initial and scattered polarization is given by $\overrightarrow{\epsilon_{0}}$ and $\overrightarrow{\epsilon^{*}}$ respectively; however, we will not consider x-ray polarization in this paper and will therefore ignore these terms.

We see immediately that all the scattering is by electrons; scattering by protons is strongly suppressed because of their large mass. Figure 2 diagrams the situation for one particular scattering site in a spherical dust grain.

Equation (11) is strictly valid only for free electrons. However, X-rays are substantially more energetic than the electronic energy bands in the solids so the electrons in the dust grain can be treated as free (Jackson, 1975, p. 683). Absorption, however, is not entirely negligible even above $1 \mathrm{keV}$ as there are a number of elements with K-shell electrons that can absorb at these energies (see $\S 3.2)$.

We can now show how to derive the limiting angle for coherent scattering. If $\left|\vec{q} \cdot \overrightarrow{r_{j}}\right| \ll 1$ in Equation (11), the exponent term reduces to unity. Then the sum is simply $N^{2} e^{2} /\left(m_{e} c^{2}\right)$, where $N$ is the total number of electrons in the dust grain. Physically, this result is due to each electron scattering independently and the resulting waves adding coherently. The intensity therefore depends upon the number of electrons in the scattering medium, and not on the spacing or density of the electrons. 
Expanding terms in $\left|\vec{q} \cdot \overrightarrow{x_{j}}\right| \ll 1$, we get, using the notation of Figure 20:

$$
\begin{aligned}
|\vec{q} \cdot \vec{r}| & =\left|\left(\frac{\omega}{c} \hat{n_{s}}-\frac{\omega_{0}}{c} \hat{n}_{i}\right) \cdot \vec{r}\right| \\
& =\left|\left(\frac{\omega}{c} \hat{n_{s}}-\frac{\omega_{0}}{c} \hat{n_{i}}\right)\right| \cdot|\vec{r}| \cdot|\cos \psi| \\
& =\left[\left(\frac{\omega}{c}\right)^{2}+\left(\frac{\omega_{0}}{c}\right)^{2}-2 \frac{\omega \cdot \omega_{0}}{c^{2}} \hat{n_{s}} \cdot \hat{n_{i}}\right]^{1 / 2} \cdot r \cdot|\cos \psi| .
\end{aligned}
$$

The complex optical constant for the dust grain relates $\omega$ and $\omega_{0}$ via $\omega=m \omega_{0}$. Substituting in (12) we get

$$
|\vec{q} \cdot \vec{r}|=k_{0}\left[m^{2}+1-2 m \cos \phi\right]^{1 / 2} \cdot r \cdot|\cos \psi|
$$

where $k_{0}=\omega_{0} / c$. The position vector $\vec{r}$ takes on all values between $0<r<a$, where $a$ is the radius of the dust, and $0<|\cos \psi|<1$. Replacing $r|\cos \psi|$ with its maximum value, $a$, the requirement that $|\vec{q} \cdot \vec{r}| \ll 1$ becomes

$$
\left|\left(m^{2}+1-2 m \cos \phi\right)\right|^{1 / 2} k_{0} a \ll 1
$$

Note that Equation (14) is equivalent to Equation (3) for $\phi \rightarrow 0$, so if the RG assumptions are met coherent scattering will occur for at least some scattering angles. We can rewrite Equation (14) using some trigonometry and the already-stated assumption that $|m-1| \ll 1$ to get a better limit on the scattering angle:

$$
2 k_{0} a \sin \left(\frac{\phi}{2}\right) \ll 1 .
$$

In the x-ray regime $k_{0} \sim 5000 E_{k e V} \mu \mathrm{m}^{-1}$. Thus $k_{0} a$ will much larger than unity for normal interstellar dust grains and $\sim 1 \mathrm{keV} \mathrm{X}$-rays, so we can use the small angle approximation for $\sin (\phi / 2)$. Waves scattered at angles where $\phi \ll 1 / k_{0} a$, when the RG assumptions are met, will have intensities proportional to $N^{2}$, the number of electrons in the grain squared.

To summarize, the RG approximation requires that the dust neither reflects photons nor significantly affects their phases. The induced scattering wave generated at the entrance to the grain must add in phase with one created at the exit. If it does not, then coherency is lost, and the amplitude of the scattered wave never becomes proportional to the number of electrons squared in the entire dust volume squared, but to a fraction of that number.

Furthermore, the combination of the RG approximation with the Drude approximation assumes that absorption is negligible at all energies. This is approximately valid when $E>1 \mathrm{keV}$, although there are astronomically abundant elements in dust with atomic energy levels above 1 $\mathrm{keV}$ are magnesium (K edge, $1.294 \mathrm{keV}$ ), aluminum (K edge, $1.550 \mathrm{keV}$ ), silicon ( $\mathrm{K}$ edge, 1.828 $\mathrm{keV}$ ), and iron ( $\mathrm{K}$ edge, $7.083 \mathrm{keV}$ ). Below $1 \mathrm{keV}$, however, there are many $\mathrm{K}$ and $\mathrm{L}$ shell edges which can absorb X-rays, an effect which will be considered in $\S 3.2$. The breakdown of the RG approximation and the effect of absorption require the use of full Mie theory to correctly calculate the intensity of the scattered light. 


\section{Mie Theory}

Mie theory calculates the exact solution for the scattering and absorption of light from a spherical particle (van de Hulst, 1957). Unlike the Rayleigh-Gans approximation, it does not assume all the scattering sites will add coherently and as a result, Mie theory can correctly calculate the scattered intensity for particles even when $a_{\mu m} \gtrsim E_{k e V}$. In the limit where $k_{0} a|m-1| \ll 1$ the Mie solution recovers the result of the Rayleigh-Gans approximation. Another advantage is that absorption can be included easily, using measured values for the index of refraction. However, although the Mie solution is exact for light scattering from a sphere, unlike the RG approximation it is not expressible in a useful analytic form.

The Mie solution requires careful numerical computation, especially for large values of the "size parameter" $x \equiv 2 \pi a / \lambda \approx 5000 a_{\mu \mathrm{m}} E_{k e V}$ where the series solution requires summation over a large number of terms to converge. We use the Mie code developed by Wiscombe $(1979,1980)$, which was written for atmospheric use but has been tested for size parameters up to 20,000, sufficient for our problem. The observed scattered intensity is obtained by integrating the intensity of the light scattered by a single dust particle to the observer, over the grain size and spatial distribution along the LOS (see eq. ( $\mathrm{A4}$ in the Appendix). These calculations can be greatly facilitated by pre-calculating tables of $(d \sigma(E, a, \phi) / d \Omega)$ for a range of energies, dust sizes, and scattering angles. [

We must point out, however, that even the Mie solution is only of an approximate nature, since dust particles are neither spherical nor compositionally homogeneous. We know that the first assumption is incorrect, since only non-spherical grains can polarize light, as grains have been observed to do (e.g. Mathis, 1990). The second assumption is particularly important, since the wavelength of a $1 \mathrm{keV}$ photon is only $1.24 \AA$, substantially smaller than the radius of any dust grain. Thus, the X-ray's path through the grain will determine which dust grain constituents it interacts with, and so the x-ray scattering will be affected by the grain's exact morphology. Exploring this possibility in detail would require a discrete dipole approximation to the dust grain constituents (e.g. Draine, 1988), which is beyond the scope of this paper.

\subsection{Comparison to the Rayleigh-Gans Approximation}

The observed halo intensity can be calculated for any x-ray energy and dust grain size, using either RG approximation or the Mie solution. Any variation in the result is due solely to the differential scattering cross section $(d \sigma / d \Omega)$ term. In Figure 3 we plot $\left(d Q_{\text {scat }} / d \Omega\right) \equiv(d \sigma / d \Omega) / \pi a^{2}$ for $2 \mathrm{keV}$ X-rays scattering from a single dust grain, as a function of the scattering angle $\phi$. The differential scattering cross section was divided by the geometrical cross section to show the

\footnotetext{
${ }^{2}$ The code needed to generate such tables, and related utilities, may be be obtained from the authors.
} 

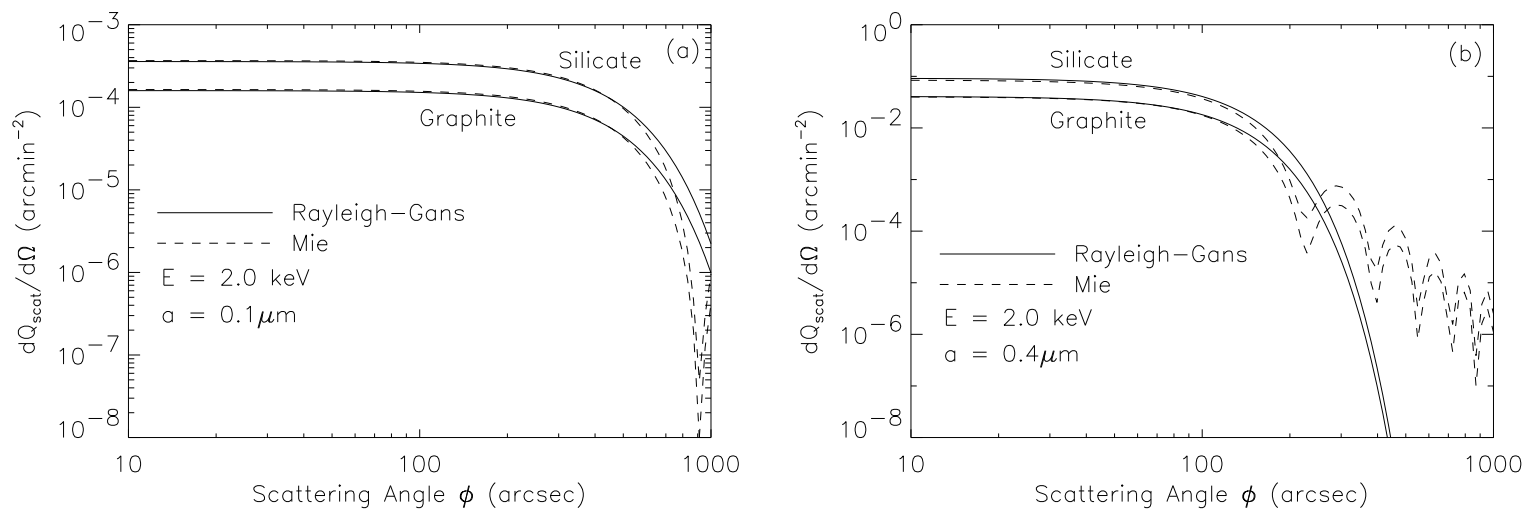

Fig. 3.- (a) The differential scattering cross sections $d Q_{s c a} / d \Omega=(d \sigma / d \Omega) / \pi a^{2}$, for a $2 \mathrm{keV}$ X-ray scattering from a $0.1 \mu \mathrm{m}$ dust grain, for both graphite and silicate dust grains. The solid lines show the Rayleigh-Gans approximation using the Gaussian approximation; the dashed lines show the equivalent Mie calculation. In this energy and $\phi$-range the two methods should give identical results; however, use of the Gaussian approximation leads to deviations at large angles. (b) Same, for $0.4 \mu \mathrm{m}$ dust grains.

relative effect of grain size on the scattering intensity. Figure 3(a) presents the results for both a graphite or silicate dust grain of radius $0.1 \mu \mathrm{m}$ and Figure 3(b) is similar but for grains of size $0.4 \mu \mathrm{m}$. The mass densities of the two types of dust, graphite and silicate, were $2.2 \mathrm{~g} \mathrm{~cm}^{-3}$ and $3.3 \mathrm{~g} \mathrm{~cm}^{-3}$ respectively. The optical constants were calculated using the Drude approximation, ignoring absorption, both for the RG and the Mie cases, so that the variation in the figures is due solely to the approximate nature of the RG solution.

Figure 3 shows that at $2 \mathrm{keV}$, the Gaussian fit to the Rayleigh-Gans approximation is adequate to the task; it deviates substantially from the Mie solution only at large angles, after the scattering intensity has dropped by at least an order of magnitude. The deviation itself is largely due to the Gaussian approximation. Using the RG approximation with the spherical Bessel function duplicates the ripples in the large-angle scattering of the $0.4 \mu \mathrm{m}$ grains and the faster drop seen for the $0.1 \mu \mathrm{m}$ grains.

When the requirement that $k_{0} a|m-1| \ll 1$ is not satified, the agreement between the RG and the Mie solution becomes substantially worse. Figure 1 is identical to Figure 3, but for X-rays of energy $0.5 \mathrm{keV}$. The "rule of thumb" suggests that in this case the RG approximation will hold for $0.1 \mu \mathrm{m}$ radius dust, but fail for the $0.4 \mu \mathrm{m}$ radius dust. Figure 1 confirms this, showing an acceptable match between the RG and the Mie calculations for the smaller grains, but showing far more total scattering with the RG approximation than in the Mie solution for the larger grain size. In this case, using the exact Bessel function solution does not help. The larger dust grain has more scattering sites, and the RG approximation assumes all the scattered waves all add 

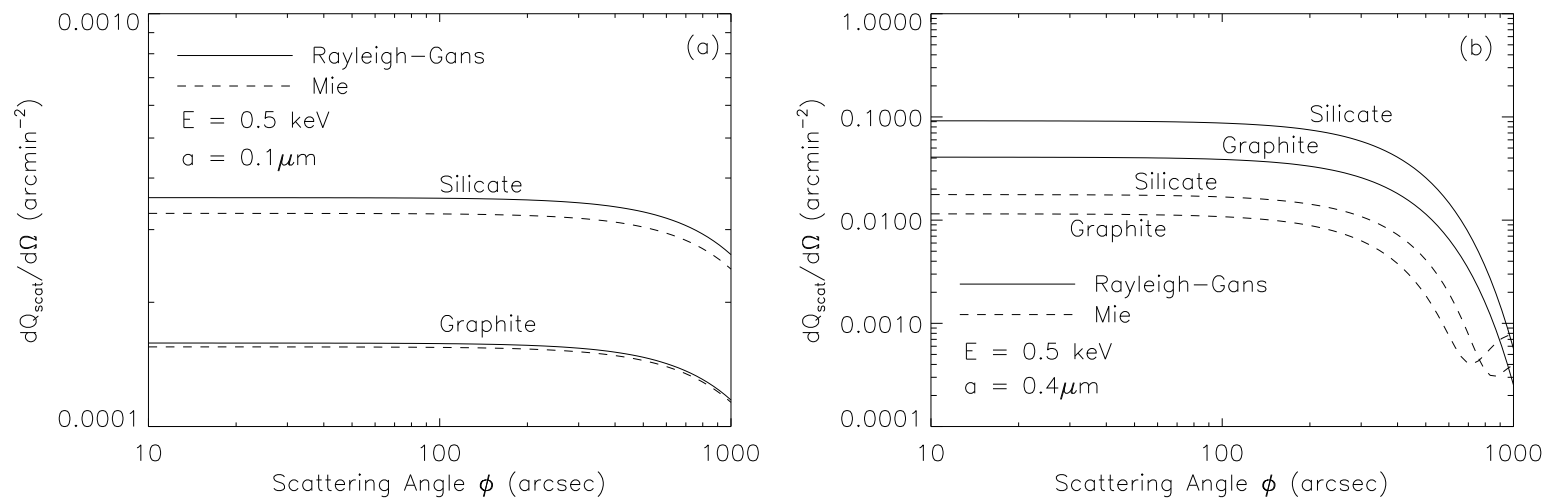

Fig. 4.- (a) Same as Figure 3, for $0.5 \mathrm{keV}$ X-rays. The RG approximation is an acceptable fit to the Mie calculations. (b) The same as (a), for a $0.4 \mu \mathrm{m}$ dust grain. The assumptions necessary for the RG approximation do not hold, and the results show that the differential scattering cross sections calculated using the Mie and RG approximation disagree substantially at all scattering angles.

coherently; the Mie solution shows that for large dust sizes this coherency assumption fails, and the scattered intensity therefore drops. Figure 4 suggests that the approximation fails abruptly, as might be predicted from the exponential nature of equation (11).

\subsection{The Effect of Absorption}

We have so far used the Drude approximation to the optical constants, ignoring any damping, in both the RG approximation and the Mie solution. Although the Drude approximation works well above $1 \mathrm{keV}$, real dust grain constituents have many $\mathrm{K}$ and $\mathrm{L}$ shell electrons with energy levels that are in the range $0.1-1 \mathrm{keV}$, and these electrons will absorb soft X-rays. The effect of absorption can be included by using the atomic scattering factor $F(E)$ (Henke, 1981) as a modifier to the Drude approximation:

$$
|m-1|=\frac{n_{e} r_{e} \lambda^{2}}{2 \pi}|F(E) / Z|
$$

where $Z$ is the atomic number of the element. However, we simply use the measured optical constants which include the absorption effects. For silicate particles $\left(\{\mathrm{MgFeSi}\} \mathrm{O}_{4}\right)$ we used the optical constants of Martin \& Rouleau (1991), and for graphite particles we used Rouleau \& Martin (1991). These values are a smooth extension of the Draine \& Lee (1984) and Laor \& Draine (1993) results into the FUV and x-ray regime. At x-ray energies, there is no distinction between graphite and amorphous carbon, so this complication need not be considered. We also considered 

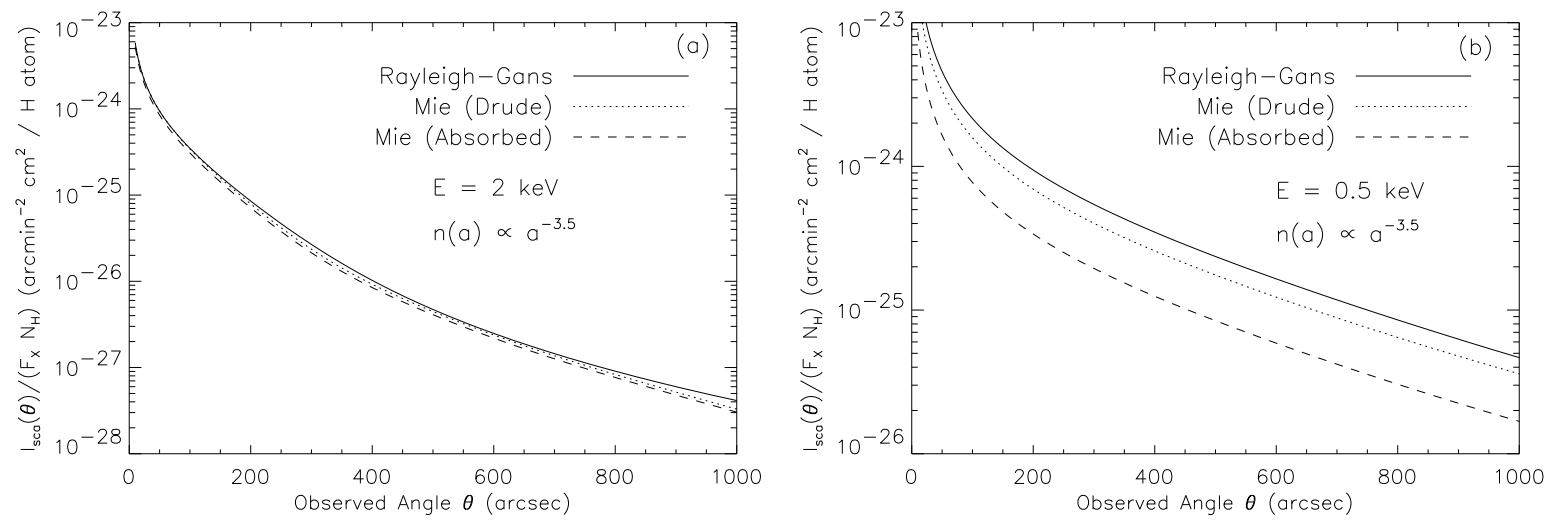

Fig. 5.- The observed halo intensity from a $2 \mathrm{keV}$ x-ray source scattering from evenly-distributed silicate and graphite dust grains with an $n(a) \propto a^{-3.5}$ size distribution. The results are shown using the RG approximation, the Mie solution with the Drude approximation to the index of refraction (i.e., without absorption) and for the Mie solution using measured optical constants.

composite dust particles as described in Mathis (1996); for this case, we used the Bruggeman rule (Bohren \& Huffman, 1983) to calculate the optical constants of the mixture.

Figure 5 (a) plots the observed halo intensity for $2 \mathrm{keV}$ X-rays and a distribution of grain sizes, calculated three different ways. We presented the observed halo, rather than showing $(d \sigma / d \Omega)$ for a single dust grain size as was shown in Figures 3 and 1 , to show the magnitude of the observable differences between the methods. For $2 \mathrm{keV} \mathrm{X}$-rays, these differences are clearly small. The halo intensity, given by equation (1), was found by integrating $(d \sigma / d \Omega)$ over an evenly distributed populates of dust particles $(f(z) \equiv 1)$ characterized by a size distribution $n(a) \propto a^{-3.5}$ with $\left\{a_{\min }, a_{\max }\right\}=\{0.005 \mu \mathrm{m}, 0.25 \mu \mathrm{m}\}$. The grains themselves consisted of a mix of silicate and graphite grains with dust to hydrogen mass ratios of 0.0057 and 0.0032 respectively.

We note that the choice of the upper limit to the size distribution, $0.25 \mu \mathrm{m}$, significantly affects the result. With this power law size distribution, most of the mass is in the largest grains. In addition, the distribution of the largest dust particles are not well-determined by extinction measurements, since when the grain size is much larger than the wavelength the extinction becomes independent of wavelength. X-ray halos are therefore a particularly useful and sensitive probe of the number and size of large dust grains. In the RG approximation the intensity is proportional to the sixth power of the radius of the dust. The Mie solution also predicts larger dust grains scatter more than small grains, although not by as large a power (see Figures 1 (a) and (b)). In general, however, the source function of the scattered intensity is heavily weighted towards large dust grains.

Figure 5 shows the cumulative effects of the approximations used in calculating the observed 
halo intensity. The simple RG approximation (with the Gaussian fit to the Bessel function and the Drude approximation to the optical constants) predicts the largest intensity. The exact Mie solution, using the Drude approximation, reduces the intensity since not all the scattered waves add coherently. The Mie solution, using actual optical constants which include absorption, has the lowest intensity as some of the impinging X-rays are absorbed in the dust.

The effect of absorption can be much larger, as shown in Figure 5(b), which is identical to Figure 5 (a) but for $0.5 \mathrm{keV}$ X-rays. The difference between the RG and the Mie solution for a realistic dust grain size distribution is quite noticeable, and Figure E(b) shows that absorption is very important at this energy. We note in passing that not all of the absorbed x-ray's energy will be deposited in the dust (Dwek \& Smith, 1996) since the ejected photo-electron may deposit only a fraction of its energy in the solid.

We have shown that the accuracy of the RG approximation is size dependent and is in general less accurate at larger grain sizes. The interstellar grain population is characterized by a mixtures of grain sizes and it is therefore interesting to apply Mie theory to an astronomical situation.

\subsection{The Energy Dependence}

In Figure 6, both the Mie and RG methods were used to calculate the intensity of scattered $\mathrm{X}$-rays from silicate grains as a function of x-ray energy, at the observed scattering angle $\theta=100^{\prime \prime}$. We see that for higher energies, the shape of the curves for the two methods begins to agree when $E \gtrsim 1 \mathrm{keV}$, but below this energy there are substantial differences. The two methods never agree exactly because of absorption, which is more important for larger grains but is not negligible even for the $0.1 \mu \mathrm{m}$ grain. The presence of absorption lines in the grains can also be seen in the halo shape, which has irregular peaks and valleys in the Mie case.

We conclude that in many astronomical situations using the RG approximation to calculate the halo intensity systematically overestimates the amount of x-ray scattering from a given dust size distribution. Consequently, the RG approximation cannot be used for x-ray energies below 1 $\mathrm{keV}$, except as an upper limit to the halo intensity. Even for energies above $1 \mathrm{keV}$, RG is only an upper limit since absorption is never totally negligible, and will reduce the total intensity of scattered light.

\section{Application and Discussion}

The total scattered light intensity will in practice depend upon the spectrum of the source $S(E)$, the spatial distribution of dust grains $f(z)$, and the grain size distribution $n(a)$. We can measure the x-ray spectrum $S(E)$, directly, and we will assume that dust grains are evenly distributed, so that $f(z) \equiv 1$. The last parameter, the dust grain size distribution $n(a)$, is not 


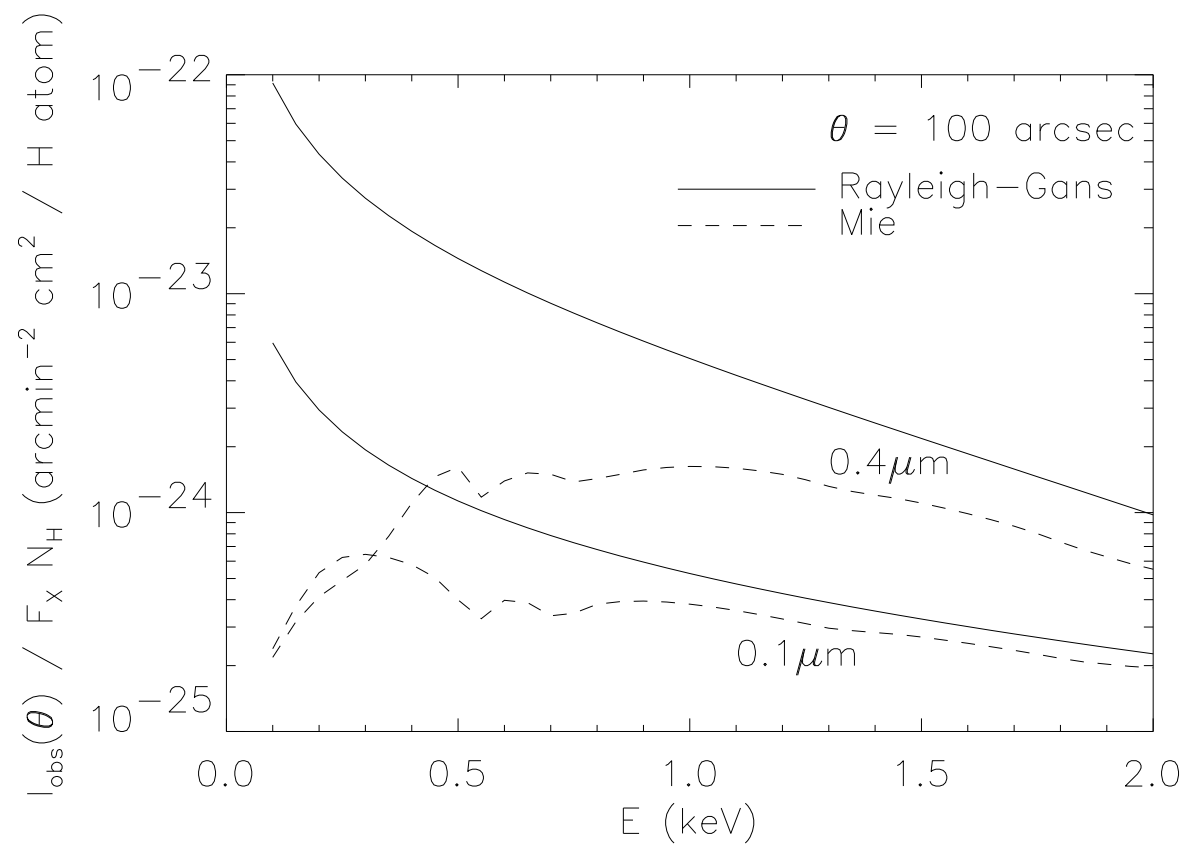

Fig. 6. - The scattered intensity at $100^{\prime \prime}$ for silicate grains of radius 0.1 and $0.4 \mu \mathrm{m}$, calculated both by the RG approximation (without absorption) and the Mie solution (including absorption) as a function of x-ray energy. For $E \gtrsim 1 \mathrm{keV}$, the two methods show the same energy dependence; below it, absorption modifies the $E$ dependence, an effect that becomes more pronounced at $0.4 \mu \mathrm{m}$.

well known, especially for larger size grains. Currently, it is measured by fitting the extinction of visible and UV starlight. We will present results using two different grain models: (1) a bare core silicate and graphite mix, with an $a^{-3.5}$ power law size distribution (following Mathis, Rumpl, \& Nordsieck, 1977)(MRN); and (2) the recent composite fluffy dust model of Mathis (1996)(M96) a distribution centered near $0.1 \mu \mathrm{m}$, consisting of composite silicate and graphite. We now apply the RG and Mie solutions with these grain models to the observed scattering from the x-ray source (Nova Cygni 1992).

\subsection{Nova Cygni 1992}

Nova Cygni 1992 exhibited a strong x-ray halo in the ROSAT PSPC (Mathis et al., 1995). The observed spectrum was formally fit with a blackbody with temperature $E=k T \sim 22 \mathrm{eV}$, absorbed by an equivalent hydrogen column density of $\mathrm{N}_{\mathrm{H}}=4.25 \times 10^{21} \mathrm{~cm}^{-2}$. Mathis et al. (1995) do not claim this is a true model of the nova, only that it is simple to calculate and fits the observed spectrum reasonably well. The observed spectrum peaked at about $0.5 \mathrm{keV}$, and with 
this choice of assumed spectrum most of the photons from the source were absorbed. However, this column density is not the best choice for use in the scattering intensity equation (1), since the value of $\mathrm{N}_{\mathrm{H}}$ is highly dependent upon the underlying true spectrum. In addition, the hydrogen column density measured from x-ray absorption depends upon the helium and metals along the LOS, and is not directly related to the number of dust grains along the LOS. Therefore, x-ray absorption measures of the hydrogen column density may not accurately reflect either the actual hydrogen column density or the dust grain column density.

The scattered halo intensity scales linearly with the column density of dust grains, which can be found by measuring the extinction along the LOS. Mathis et al. (1995) (and references therein) estimated the reddening $\mathrm{E}(\mathrm{B}-\mathrm{V})$ to be between 0.19-0.31 mag. Using the standard extinction law value of $R=3.1$, this translated into a hydrogen column density in the range $\mathrm{N}_{\mathrm{H}}=1.1-1.8 \times 10^{21} \mathrm{~cm}^{-2}$. There is a significant difference between the x-ray and extinction derived hydrogen column densities. The former depends upon the source spectrum over a broad range of energies, whereas the latter depends on the source spectrum over a much narrower range (B and $\mathrm{V}$ bands), and provides a more direct measurement of the dust column density along the LOS. We therefore prefer the extinction determined hydrogen column density, and use a value of $\mathrm{N}_{\mathrm{H}}=1.45 \times 10^{21} \mathrm{~cm}^{-2}$. For the Nova Cygni source Mathis et al. (1995) noted that this column density creates only a small optical depth for scattering, so the assumption of single-scattering is adequate. Nonetheless, the halo was clearly visible in the data to angles greater than $800^{\prime \prime}$ before it merged with the background.

Mathis et al. (1995) fitted the halo using the RG approximation for the differential scattering cross section and found that an MRN model with a column density chosen to produce the observed visual extinction would scatter more X-rays than were seen. Noting that the intensity of the scattered light is proportional to the grain density squared, they proposed a model consisting of composite fluffy dust (CFD) particles with substantial amounts of vacuum which would scatter fewer X-rays but would nevertheless produce the same observed visual extinction. The amount of light scattered from a fluffy grain is less than that from the same size dense grain, since the fluffy grain has fewer electrons with which to scatter the X-rays. This reduction in scattering remains even when the total amount of mass in dust is constant, since the intensity is proportional to the number of electrons in each grain squared. Therefore, having twice as many grains which are 50\% vacuum will lead to a net $50 \%$ drop in the intensity-when the RG approximation is valid.

In the $0.5 \mathrm{keV}$ energy range, however, the $\mathrm{RG}$ approximation is not valid for dust grains larger than $\sim 0.1 \mu \mathrm{m}$, for two reasons. First, the scattered waves will not add coherently as the dust grain material will affect their phases substantially, and secondly, absorption in the dust grain will remove some of the $\mathrm{X}$-rays altogether. As a result, using the $\mathrm{RG}$ approximation with the no-damping Drude approximation to the optical constants will tend to substantially overpredict the amount of scattered light and so the Mathis et al. (1995) result must be re-evaluated using the Mie solution. 
Table 1: Dust grain parameters

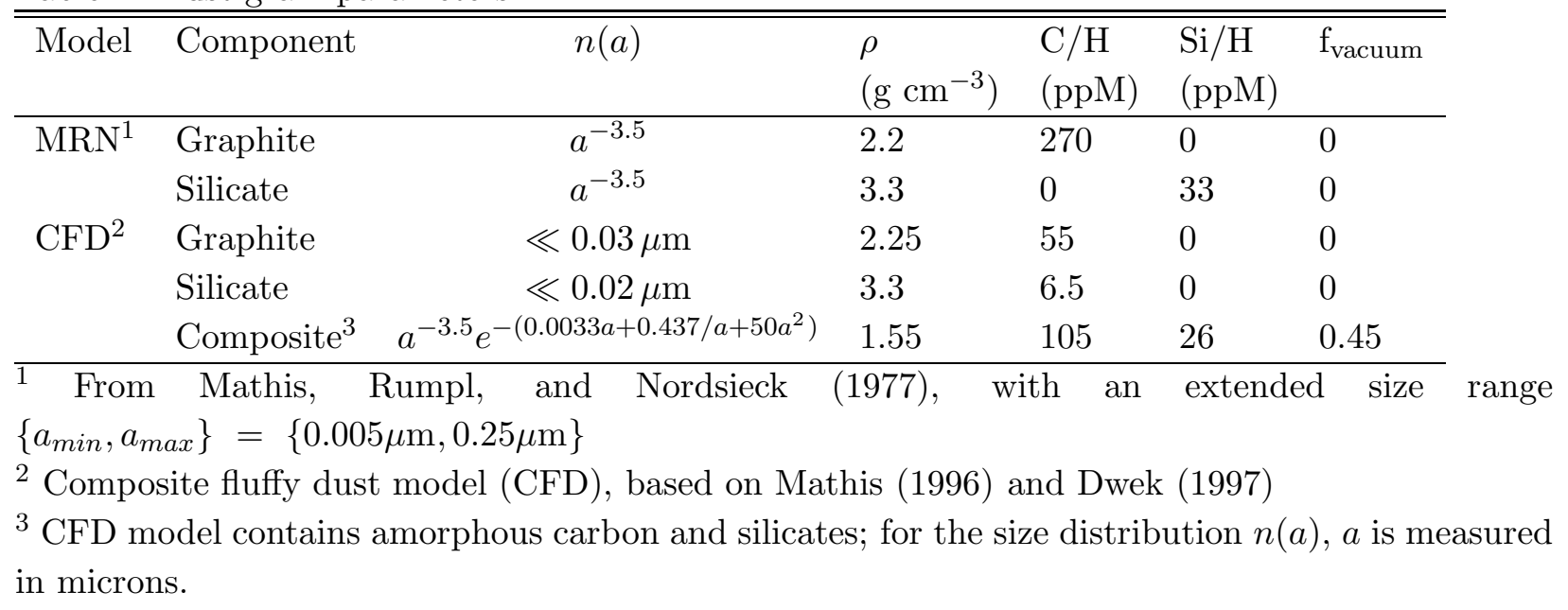

To examine the robustness of the conclusions derived by Mathis et al. (1995) for the Nova Cygni data, we modeled the same data using Mie theory for the standard MRN model, and the newly-proposed CFD model (M96). The parameters of the two models are presented in Table (1. The two dust models produce an overall equally good fit to the average interstellar extinction curve (Dwek, 1997). However, the CFD model produces a visual extinction that is $6.7 \%$ higher than the MRN model, so we normalized the x-ray scattering intensity downward by the same amount. With this renormalization the two models produce the same amount of visual extinction towards the x-ray source. We limited our results to the case of evenly distributed dust, i.e. $f(z) \equiv 1$.

Our results are shown in Figure 7, which shows observed halo intensity as well as the intensity calculated using the RG approximation and the Mie solution, for both the fluffy and the bare-core grain models. For both types of grains, the RG approximation overestimates the amount of scattering; in this case, neither the MRN or the Mathis (1996) composite dust fits the data. However, both models, when calculated using the Mie solution, predict similar amounts of scattering that are slightly less than the observed scattering. While none of the four models fits the data particularly well, we can make a number of useful observations:

1. The fit depends on $\mathrm{N}_{\mathrm{H}}$. The measured column density was $\mathrm{N}_{\mathrm{H}}=1.45 \pm 0.35 \times 10^{21} \mathrm{~cm}^{-2}$. Figure 07 uses the central value, but using the upper $1 \sigma$ value would mean the Mie solution models fit the data well. To fit the RG approximation with fluffy dust requires the lower $1 \sigma$ value, and an even larger value for the dense dust.

2. While there is a large difference between the fluffy and dense dust models in the RG solution, the difference is much smaller when using the Mie calculations. The likely reason for this is that for a given dust size, absorption is more important for the dense dust than for the fluffy dust, and as a result the scattering from dense dust is reduced relative to fluffy dust. Therefore, we conclude that the Nova Cygni 1992 data does not distinguish between fluffy 


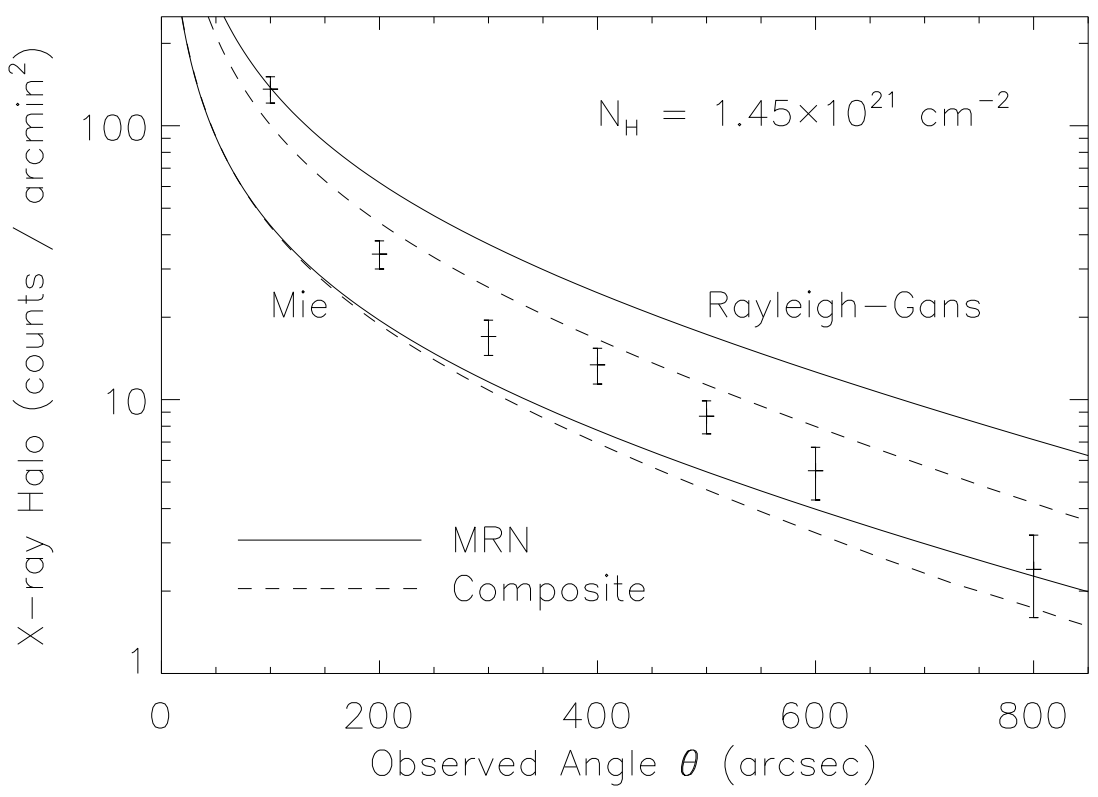

Fig. 7.- Nova Cygni 1992 data, with MRN (bare spherical silicate and graphite grains) and composite fluffy dust models (Mathis, 1996). The RG approximation overestimates the amount of scatter in both cases, while the Mie solution underestimates the data, with similar results for either type of dust model. Unlike the RG approximation results, the Mie solution does not distinguish between fluffy and dense dust. This figure illustrates that the Nova Cygni 1992 data cannot be used to infer the morphology of the scattering dust.

and dense dust.

Finally, it is important to note that while the Mie solution models marginally fit the data at larger angles, they substantially underestimate the observed scattering at $100^{\prime \prime}$. At small observed angles $\theta$, dust along the LOS in the vicinity of the source dominates the observed intensity. Therefore, the larger halo intensity seen at small angles may be due to an increased number of dust grains near the source.

\section{Conclusions}

$\mathrm{X}$-ray scattering is a powerful tool for measuring the composition and size distribution of interstellar dust. However, many approximations and assumptions are necessary to create models of the data. We have assumed, for example, that dust grains are spherical, homogeneous, and evenly distributed throughout the ISM, assumptions whose effects should be tested. We have explored the use of the Rayleigh-Gans approximation for the differential scattering cross section, 
Table 2: Nova Cygni 1992 Scattering Intensity: Data (from Mathis et al. (1995)) and Models

\begin{tabular}{llllll}
\hline \hline$\theta$ (arcsec) & Observed & MRN RG & MRN Mie & Composite RG & Composite Mie \\
\hline 0 & $6.62 \times 10^{4}$ & & & & \\
100 & $136 \pm 15$ & 137.7 & 43.2 & 100.5 & 42.9 \\
200 & $34 \pm 4$ & 62.0 & 19.5 & 44.2 & 18.7 \\
300 & $17 \pm 2.5$ & 36.9 & 11.6 & 25.7 & 10.9 \\
400 & $13.4 \pm 2.0$ & 24.6 & 7.73 & 16.6 & 6.94 \\
500 & $8.7 \pm 1.2$ & 17.3 & 5.45 & 11.3 & 4.68 \\
600 & $5.5 \pm 1.2$ & 12.6 & 3.98 & 7.99 & 3.26 \\
800 & $2.4 \pm 0.8$ & 7.14 & 2.27 & 4.19 & 1.73 \\
\hline
\end{tabular}

which when combined with the Drude approximation to the index of refraction provides a very convenient analytical form for discussing x-ray scattering. We find that the RG approximation works well for normal interstellar dust parameters and energies at or above $2 \mathrm{keV}$, but below this energy it overestimates the halo intensity.

We re-examined the Nova Cygni 1992 data, which has a strong x-ray halo, and found that it does not distinguish between fluffy and dense dust. Mie calculations using measured optical constants produce equally good fits for the standard MRN model, and for fluffy dust particles. Consequently the Nova Cygni data cannot be used as evidence that interstellar dust particles are fluffy, though they may be.

The future of x-ray scattering is bright. Although the ROSAT PSPC has the highest energy-resolved spatial resolution available currently, the AXAF CCD Imaging Spectrometer (ACIS) and High-Resolution Camera (HRC) detectors will have arcsecond resolution with low to moderate energy resolution. Clearly, nearly every point source seen with AXAF will include an $\mathrm{x}$-ray halo of some kind. The high angular resolution will allow observers to extract halos down to $10^{\prime \prime}$ or less, where the scattered intensity is due almost entirely to grains very near the source. This will be a bonanza for dust grain theorists, as it will provide a new handle on the size distribution of larger grains as well as the position-dependence of grain populations. At the same time it will add a difficulty to the data analysis of diffuse sources, since a calculation of the x-ray halo due to dust scattering will have to made in order to remove it, in a process similar to the "dereddening" required of optical and UV measurements.

\section{Acknowledgments}

We thank Alain Léger for invoking our interest in this problem. This work was performed while one of the authors (RKS) held a National Research Council-GSFC Research Associateship. This research was also supported by NASA Astrophysics Theory Program grant \#344-02-05-01. 


\section{A. Deriving the Halo Intensity}

Consider a dust particle of size $a$ at a point $\mathrm{P}$, illuminated by an x-ray source $(\mathrm{S})$ a distance $R_{1}$ away, which scatters photons (of energy $E$ ) towards an observer $(\mathrm{O})$ at a distance $R_{2}$, as is shown in Figure 1. Given a source luminosity of $L(E)$, the flux at $\mathrm{P}$ is $L(E) \exp \left(-\mathrm{N}_{\mathrm{H}}(\mathrm{SP}) \sigma(E)\right) / 4 \pi R_{1}^{2}$, where $\mathrm{N}_{\mathrm{H}}(\mathrm{SP})$ is the column density along the line SP and $\sigma(E)$ is the average interstellar absorption cross section at energy $E$. Then the luminosity of scattered photons into solid angle $d \Omega$ is

$$
L_{\text {scat }}=\frac{L \exp \left\{-\sigma(E) \mathrm{N}_{\mathrm{H}}(\mathrm{SP})\right\}}{4 \pi R_{1}^{2}}\left(\frac{d \sigma}{d \Omega}\right) d \Omega .
$$

Now consider the photons arriving at the observer, who measures the flux of photons from the scattered dust particle, using a detector of size $d A^{\prime}$, and a telescope with opening angle $d \Omega^{\prime}$. Then we can choose $d \Omega$ so that $d A^{\prime}=R_{2}^{2} d \Omega$, and the observer obtains a photon count rate (with the opening angle $d \Omega^{\prime}$ for the telescope) of

$$
C=\frac{L \exp \left\{-\sigma(E) \mathrm{N}_{\mathrm{H}}(\mathrm{SP})\right\}}{4 \pi R_{1}^{2}}\left(\frac{d \sigma}{d \Omega}\right) \exp \left\{-\sigma(E) \mathrm{N}_{\mathrm{H}}(\mathrm{PO})\right\} \frac{d A^{\prime}}{R_{2}^{2}}
$$

This is the result for a single dust particle; we can now integrate it over all dust particles in the scattering volume. Assume that there are $n_{g}(P)$ dust grains per unit volume at position $\mathrm{P}$. We will integrate over the line of sight using a differential volume element with length $d R_{2}$ and solid angle $d \Omega^{\prime}$. The volume is $d V=R_{2}^{2} d R_{2} d \Omega^{\prime}$, and we obtain the total count rate by integrating over $R_{2}$ :

$$
\begin{aligned}
C_{t o t} & =\int \frac{L \exp \left\{-\sigma(E) \mathrm{N}_{\mathrm{H}}(\mathrm{SP})\right\}}{4 \pi R_{1}^{2}}\left(\frac{d \sigma}{d \Omega}\right) n_{g}(P) \exp \left\{-\sigma(E) \mathrm{N}_{\mathrm{H}}(\mathrm{PO})\right\} \frac{d A^{\prime}}{R_{2}^{2}} R_{2}^{2} d l d \Omega^{\prime} \\
& =d A^{\prime} d \Omega^{\prime} \int \frac{n_{g}(P) L \exp \left(-\left\{\sigma(E)\left[\mathrm{N}_{\mathrm{H}}(\mathrm{SP})+\mathrm{N}_{\mathrm{H}}(\mathrm{PO})\right]\right\}\right.}{4 \pi R_{1}^{2}}\left(\frac{d \sigma}{d \Omega}\right) d R_{2} .
\end{aligned}
$$

Thus the count rate (in photons per second) in a detector with area $d A^{\prime}$ and opening angle $d \Omega^{\prime}$ is given by $C_{t o t}$. We can therefore write the intensity of the scattered light as

$$
I_{s c a}(E, a, \theta)=\int \frac{n_{g}(P) L \exp \left\{-\sigma(E)\left[\mathrm{N}_{\mathrm{H}}(\mathrm{SP})+\mathrm{N}_{\mathrm{H}}(\mathrm{PO})\right] \sigma(E)\right\}}{4 \pi R_{1}^{2}}\left(\frac{d \sigma}{d \Omega}\right) d R_{2} .
$$

This equation must then be integrated over a energy bandpass and a range of dust size and position distributions. In general, the function $n_{g}$ will be a function of dust radius $a$ as well as a function of position. Therefore, the total scattering for a given energy range $\left\{E_{1}, E_{2}\right\}$ will be

$$
I_{s c a}(\theta)=\int_{E_{1}}^{E_{2}} \int_{a_{\min }}^{a_{\max }} \int_{0}^{D} \frac{n_{g}(a, P) L(E) \exp \left\{-\sigma(E)\left[\mathrm{N}_{\mathrm{H}}(\mathrm{SP})+\mathrm{N}_{\mathrm{H}}(\mathrm{PO})\right]\right\}}{4 \pi R_{1}^{2}}\left(\frac{d \sigma}{d \Omega}\right) d R_{2} d a d E .
$$

This equation is completely general; however, we can simplify it substantially if the observed angle $\theta$ is very small, limiting the observations to very small angles. In this case, the path traveled 
by a scattered photon will be very close to that traveled by an unscattered photon and the total hydrogen column density for the source, $\mathrm{N}_{\mathrm{H}}(\mathrm{SO})$, will be nearly the same as that of the scattered photons, $\mathrm{N}_{\mathrm{H}}(\mathrm{SP})+\mathrm{N}_{\mathrm{H}}(\mathrm{PO})$. As a result, we have the following approximation:

$$
\frac{L(E) \exp \left\{-\sigma(E)\left[\mathrm{N}_{\mathrm{H}}(\mathrm{SP})+\mathrm{N}_{\mathrm{H}}(\mathrm{PO})\right]\right\}}{4 \pi R_{1}^{2}} \approx \frac{F_{X}(E) \cos (\phi-\theta)}{(1-z)^{2}}
$$

where $F_{X}(E)$ is the observed flux from the source. We can then rewrite equation (A4) in terms of the normalized projected position coordinate $z$ and the scattering angles $\theta$ and $\phi$ :

$$
I_{s c a}\left(E_{b}, \theta\right)=\int_{E_{b}} \int_{a_{\min }}^{a_{\max }} \int_{0}^{1} \frac{n_{g}(a, z, \theta) F_{X}(E) \cos (\phi-\theta)}{(1-z)^{2}}\left(\frac{d \sigma}{d \Omega}\right) \frac{D}{\cos \theta} d z d a d E
$$

We can reduce this further by assuming that the dust size and position distribution is separable, and is independent of the hydrogen density $n_{H}$, so that

$$
n_{g}(a, z, \theta) d a=n(a) f(z) n_{H}(z) d a .
$$

We have used the assumption of similar paths for the scattered and direct photons here as well. Using these, and assuming $\cos \theta \approx 1$, we can rewrite (A6), to get

$$
I_{s c a}(\theta)=\mathrm{N}_{\mathrm{H}} \int_{E_{1}}^{E_{2}} F_{X}(E) \int_{a_{\min }}^{a_{\max }} n(a) \int_{0}^{1} \frac{f(z) \cos (\phi-\theta)}{(1-z)^{2}}\left(\frac{d \sigma(E, a, \phi)}{d \Omega}\right) d z d a d E
$$

where $\mathrm{N}_{\mathrm{H}} \equiv \int n_{H}(z) D d z$ is the hydrogen column density to the source. The final assumption necessary to derive equation (11) in the text is that $\cos (\phi-\theta) \approx 1$. We have already limited the observed angle $\theta$ to small angles. Although close to the x-ray source $\phi$ can be large even for very small values of $\theta,(d \sigma / d \Omega)$ is strongly suppressed at scattering angles above $1^{\circ}$, so that in practice, all the contribution to the scattered light comes from scattering angles below $1^{\circ}$. Therefore the actual scattering angle $\phi$ will be small, so $\cos (\phi-\theta) \approx 1$. Note also that $\phi>\theta$ for all geometries. For a nonuniform dust distribution, in which a significant amount of dust is near the source the contribution from larger scattering angles may be important, and an exact expression for $\cos (\phi-\theta)$ will then be required.

\section{REFERENCES}

Anders, E. \& Grevesse, N. 1989, Geochimica et Cosmochimica Acta, 53, 197

Bohren, C. F. \& Huffman, D. R. 1983, Absorption and Scattering of Light by Small Particles, New York: Wiley

Canizares, C. R 1990, AdSpR, 10, 261

Cardelli, J. A., Meyer, D. N., Jura, M., \& Savage, B. D. 1996, ApJ, 467, 334

Draine, B. T. 1988, ApJ, 333, 848 
Draine, B. T. \& Lee, H. M. 1984, ApJ, 285, 89

Dwek, E., et al. 1997, ApJ, 475, 565

Dwek, E. 1997, ApJ, 484, 779

Dwek, E. \& Smith, R. K. 1996, ApJ, 459, 686

Glatter, O., \& Kratky, O. 1982, "Small Angle X-ray Scattering," London: Academic Press

Henke, B. L. 1981, in Low Energy X-Ray Diagnostics, ed. D. T. Attwood and B. L. Henke (New York: AIP), p. 146

Jackson, J. D., 1975, "Classical Electrodynamics", 2nd ed. New York: Wiley

Laor, A. \& Draine, B. T. 1993, ApJ, 402, 441

Martin, P. G. \& Rouleau, F. 1991, in Extreme Ultraviolet Astronomy, ed. R. F. Malina \& S. Bowyer, p. 341.

Mathis, J. S., Rumpl, W., \& Nordsieck, K. H. 1977, ApJ, 217, 425 (MRN)

Mathis, J. S. 1990, ARA\&A, 28, 37

Mathis, J. S. \& Lee, C. W. 1991, ApJ, 376, 490 (ML91)

Mathis, J. S., Cohen, D., Finley, J. P., \& Krautter, J. 1995, ApJ, 449, 320.

Mathis, J. S. 1996, ApJ, 472, 643 (M96)

Mauche, C. W. \& Gorenstein, P. 1986, ApJ, 302, 371 (MG96)

Mauche, C. W. \& Gorenstein, P. 1989, ApJ, 336, 843

Overbeck, J. W. 1965, ApJ, 141, 864

Predehl, P. \& Schmitt, J. H. H. M. 1995, A\&A, 246, L40

Predehl, P. \& Klose, S. 1996, A\&A, 306, 283 (PK96)

Predehl, P. 1996, in Röntgenstrahlung from the Universe, MPE Report 263, p. 623

Rouleau, F. \& Martin, P. G. 1991, ApJ, 377, 526

van de Hulst, H. C. 1957, Light Scattering by Small Particles (New York: Dover)

Wiscombe, W. 1979, "Mie Scattering Calculations-Advances in Technique And Fast, Vector-Speed Computer Codes", NCAR Tech Note TN-140+STR, National Center For Atmospheric Research, Boulder, Colorado

Wiscombe, W. 1980, Appl. Opt. 19, 1505

Woo, J. W., Clark, G. W., Day, C. S. R., Nagase, F., \& Takeshima, T. 1994, ApJ, 436, L5 